\title{
Assessment of the radiative properties of some semi-conductors for applications in thermophotovoltaic and thermophotonic conversion systems
}

\author{
Fabrice Kwefeu Mbakop ${ }^{1}$, Noël Djongyang ${ }^{1 *}$, Joseph Yves Effa $^{2}$, Danwé Raïdandi ${ }^{1,3}$, \\ Jean Luc Dit Bouerdjila Nsouandélé ${ }^{1}$, René Tchinda ${ }^{4}$ \\ ${ }^{1}$ Department of Renewable Energy, The Higher Institute of the Sahel, University of Maroua, PO Box 46 Maroua, Cameroon \\ ${ }^{2}$ Department of Physics, Faculty of Science, University of Ngaoundere, PO Box 454 Ngaoundere, Cameroon \\ ${ }^{3}$ Department of Mechanical Engineering, National Advanced Polytechnic School, University of Yaounde I, \\ PO Box 8390 Yaounde, Cameroon \\ ${ }^{4}$ LISIE, Fotso Victor University Institute of Technology, University of Dschang, PO Box 134 Bandjoun \\ *Corresponding author E-mail: noeldjongyang@gmail.com
}

Copyright $\odot 2014$ Mbakop et al. This is an open access article distributed under the Creative Commons Attribution License, which permits unrestricted use, distribution, and reproduction in any medium, provided the original work is properly cited.

\begin{abstract}
Today, major energy challenge is to find alternatives to exhaustible and polluting fossil fuels. Photovoltaic (PV), thermophotovoltaic (TPV) and thermophotonic (TPX) systems offer interesting prospects for the purpose. However, these technologies require a judicious choice of the emitting materials. This paper presents an assessment of the radiative properties of some semi-conductors that can be used for these systems. The method is based on the principles of thermal radiation, Fresnel's theories of radiation, and Maxwell's equations describing the classical theory of the propagation of electromagnetic waves in a homogeneous and isotropic medium. The influence of the extinction index on the optical characteristics of some materials $(\mathrm{SiC}, \mathrm{Si}, \mathrm{Ge}$, and $\mathrm{ZnO})$ is evaluated. The transfer matrix method was used to evaluate and assess the behaviour of the radiative properties of strongly and weakly absorbing multilayered structures. It was found that the use of a non-reflecting treatment (glass or $\mathrm{ZnO}$ ) on the material, enables to reduce multiple reflections and therefore to improve the efficiency of the system.
\end{abstract}

Keywords: Semi-Conductors; Thermal Radiation; Energy Conversion Systems; Photovoltaic; Thermophotovoltaics; Thermophotonics.

\section{Introduction}

The ever-increasing demand for conventional energy sources like coal, natural gas and crude oil is driving society towards the research and development of alternate energy sources. TPV and TPX systems offer interesting prospects for the purpose. However, these technologies require a judicious choice of the emitting material. Thermophotovoltaics involves the photovoltaic conversion of radiation from an emitter heated by various sources including sunlight. The main difference from the normal solar photovoltaics is that, the emitted energy unable to be used by the receiver can be recycled allowing high conversion efficiency. Thermophotonics is a recent development of this concept. Here, the emitter (heated diode) is "active", increasing therefore the rate of energy transfer for a given emitter temperature and concentrating the emission in an energy range more suited for conversion by the cell [1], [2].

Few works related to TPV and TPX systems are found in the literature. These essential engineering disciplines include areas such as heat and mass transfer, optics with infrared filters, high temperature materials with ceramics, incandescent lamp with vacuum designs and radiant burners [2]. Green [5] was the first to propose publicly in a forum, works on TPX in 2000. Nowadays, no TPX device is known to have been designed because of the difficulty of operation at very high temperature with sufficient efficiency [3], [5]. Most research groups focus on cells from Gallium Antimonide (GaSb), Silicon ( $\mathrm{Si}$ ), and Gallium Arsenide Indium (InGa-As). The germanium (Ge) is also cost effective [6].

The main element in the TPV and TPX conversion system is the selective emitter i.e. a heat source which emit very intensely in a given spectral band closed to the gap of the photosensitive material, and not elsewhere [8]. Most of the 
research and development in TPV and TPX systems are related to methods of controlling the properties of the emitter which is very important to the overall efficiency of the system. Drevillon et al [7] used genetic algorithms to propose some solutions, but the resulting structures are too complex since they consist of many layers. Indeed, it was recently shown that one can have very different thermal radiation from that of a black element by controlling it spectrally and directionally [9]. Thus the importance of the theory of electromagnetic waves is incontestable in order to obtain the specific optical properties of the material at the interface (transmitter). The energy transfer between the components of TPV or TPX system is mainly made up of thermal radiation which propagates in the form of electromagnetic waves to a PV cell [10]. For an ideal case of TPV and TPX systems, it is necessary to work on the properties of emission sources and suitable materials for these systems.

In this paper, we are investigating into the radiative properties of some semi-conductors for TPV and TPX applications. The method is based on the principles of thermal radiation, Fresnel's theories of radiation, and Maxwell equations describing the classical theory of the propagation of electromagnetic waves in homogeneous and isotropic media.

\section{Modeling of thermal radiation in a multilayered material}

\subsection{Optical properties of thermal radiation}

The optical properties of materials are governed by three main phenomena: transmission, reflection and absorption (figure 1). These phenomena are respectively characterized by a transmittance or transmission factor (T), a reflectance or reflection factor (R), an absorbance or absorption factors (A) [11]. These parameters are important for TPV and TPX systems. They help to evaluate and control the electromagnetic waves or the incident light, so that to get a maximum emissivity, which will be emitted toward the TPV or TPX cell.

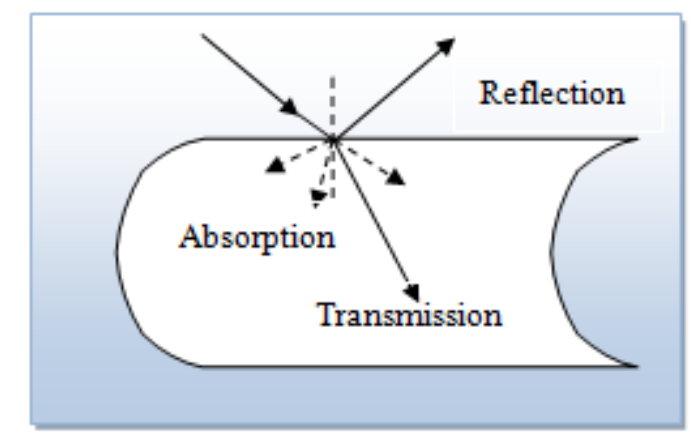

Fig. 1: Reflection, Transmission and Absorption of Radiation [12].

A thermal radiation is defined by some fundamental laws. The spectral radiance ${ }^{L_{\lambda}^{0}}(\lambda, T)$ of the black element radiation is isotropic and given by Planck's law as follows [13]:

$M_{\lambda}^{0}(\lambda, T)=\pi L_{\lambda}^{0^{\prime}}(\lambda, T)=\frac{2 \pi h_{p} \lambda^{-5}}{\exp \left(h_{p} c / k_{B} \lambda T\right)-1}$

The total luminance is obtained by integrating through the entire wavelengths of the monochromatic luminance; and the Stephan-Boltzmann's law is obtained [13]:

$L^{0}=\int_{0}^{\infty} \frac{2 \cdot \pi \cdot h \cdot c^{2} \cdot \lambda^{-5}}{\exp \left(h \cdot c / k_{B} \cdot \lambda T\right)-1} d \lambda=\sigma T^{4}$

Taking into account Wien's theory related to the monochromatic luminance of a black body, Planck's formula can be approximated by [14]:

$M_{\lambda}^{0}=2 \cdot \pi \cdot c^{2} \cdot \lambda^{-5} \cdot \exp \left(-h \cdot c / k_{B} \cdot \lambda T\right)$

The first law of Wien related to thermal radiation is given by [14]:

$\lambda_{\text {max }} T=2898 \mu m . K=c s t$

While the second law of Wien is [14]:

$M_{\lambda_{m}}^{0}=1.287 .10^{-5} T^{5} W \cdot m^{-3}$

Kirchhoff's law describing the relationship between the emission and absorption is given by [15]:

$\varepsilon(\lambda, \theta, \varphi)=\alpha(\lambda, \theta, \varphi)$ 
Where $\varepsilon(\lambda, \theta, \varphi)$ are the monochromatic directional emissivity and $\alpha(\lambda, \theta, \varphi)$ the monochromatic directional absorptivity? This law is valid only if one assumes local thermodynamic equilibrium. If the surface is in thermodynamic equilibrium, the Kirchhoff's law can be used and we have [15]:

$1=r(\lambda, \theta, \varphi)+\tau(\lambda, \theta, \varphi)+\varepsilon(\lambda, \theta, \varphi)$

For opaque surfaces, $\tau=0$ thus:

$\varepsilon(\lambda, \theta, \varphi)=1-r(\lambda, \theta, \varphi)$

\subsection{Studied model}

Figure 2 shows the model used to study the radiative properties (reflection, transmission, absorption) of the semiconductors in the case of high and low absorption.

\begin{tabular}{|cc|}
\hline $\mathrm{n}_{0}$ & $\begin{array}{c}\text { Air } \\
\text { (air layer) }\end{array}$ \\
\hline $\mathrm{n}_{1 .}$ & Selective emitter \\
& (semi-conductor layer) \\
\hline
\end{tabular}

Fig. 2: Studied Model.

The radiative properties of the structure are evaluated by the transfer matrix method. This method is based on the propagation of the electromagnetic field in an optically isotropic homogeneous structure and modelled by Maxwell's relations. It helps to understand the usefulness of the antireflection layer that allows the reduction of losses due to reflection.

\section{Transfer matrix method}

The propagation of the electromagnetic field within a homogeneous optically and isotropic structure is described by the Maxwell's relations. The following equation called D'Alembert is used [16]:

$\Delta \stackrel{\mathrm{r}}{E}-\mu_{0} \cdot \mu_{r} \cdot \sigma \frac{\partial{ }^{\mathrm{r}}}{\partial t}-\mu_{0} \cdot \mu_{r} \cdot \varepsilon_{0} \cdot \varepsilon_{r} \frac{\partial^{2} \stackrel{\mathrm{r}}{E}}{\partial^{2} t^{2}}=\stackrel{\mathrm{r}}{0}$

The wavelength in the vacuum $\lambda=2 . \pi . c / \omega$ of frequency $v=\omega / 2 . \pi$ and with a wave vector $k$ directed along the direction of propagation is defined such that $(\stackrel{\Gamma}{E}, \stackrel{\Gamma}{H} \stackrel{k}{k})$ is a direct trihedral. $\mathrm{K}$ is linked to the pulsation by the relation: $k=n \omega / c$

The multilayered system shown in figure 3 is annotated by the components of the electric field at each interface. For the sake of convenience, this figure is oriented such that the incident waves propagate from the left to the right. The ' + ' and '-' are used to distinguish the incident waves to reflected one. Signs (') are used to distinguish the waves that are at the right side of an interface from those of the left side.

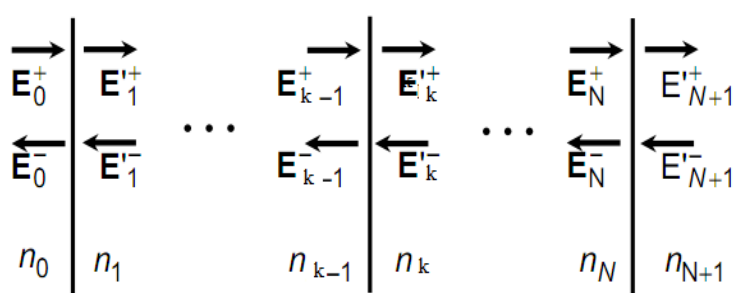

Fig. 3: A Multi-layered Structure Used to Illustrate the Principle of the Matrix Transfer Method.

The multi-layered structure is made of $\mathrm{N}+1$ interfaces and $\mathrm{N}$ layers. The layer of the extreme left side is assumed to be air $(\mathrm{n} 0=1)$ and the far right the substrate. The boundary conditions for the vectors of the electric field $\mathrm{E}$ at each side of any interface allows a simple description by a $2 \times 2$ matrix. Thus, for the kth interface, the relationship between the components of the field is given by:

$\left(\begin{array}{c}E_{k-1}^{+} \\ E_{k-1}^{-}\end{array}\right)=D_{k-1, k}\left(\begin{array}{c}E_{k}^{,+} \\ E_{k}^{,-}\end{array}\right)$ 
Where $D_{k-1, k}=\frac{1}{t_{k-1, k}}\left(\begin{array}{lr}1 & r_{k-1, k} \\ r_{k-1, k} & 1\end{array}\right)$

The field components at the left and right sides of the k-th layer are defined by the propagation matrix Pk: $\left(\begin{array}{c}E_{k}^{,+} \\ E_{k}^{,-}\end{array}\right)=p_{k}\left(\begin{array}{c}E_{k}^{+} \\ E_{k}^{-}\end{array}\right)$

With $P_{k}=\left(\begin{array}{ll}\exp \left(i . \varphi_{k}\right) & 0 \\ 0 & \exp \left(-i . \varphi_{k}\right)\end{array}\right)$

$\varphi_{k}=\frac{2 \cdot \pi \cdot n_{k} \cos \left(\theta_{k}\right)}{\lambda} d_{k}$ Represents the dephasing for $\theta \neq 0$

$\lambda$ is the wavelength in the medium.

For k layers, the Fresnel coefficients are written:

For a parallel polarization:

$r_{k}^{s}=\frac{E_{k-1, r}^{s}}{E_{k-1, i}^{s}}$ And $t_{k}^{s}=\frac{E_{k, i}^{s}}{E_{k-1, i}^{s}}$

For a perpendicular polarisation:

$r_{k}^{p}=\frac{E_{k-1, r}^{p}}{E_{k-1, i}^{p}}$ And $t_{k}^{p}=\frac{E_{k, i}^{p}}{E_{k-1, i}^{p}}$

These coefficients are expressed as functions of the refractive indexes nk-1 and nk of the layers using Fresnel laws as follows:

$\begin{array}{ll}r_{k}^{s}=\frac{n_{k-1} \cos \left(\theta_{k}\right)-n_{k} \cos \left(\theta_{k-1}\right)}{n_{k-1} \cos \left(\theta_{k}\right)+n_{k} \cos \left(\theta_{k-1}\right)} & t_{k}^{s}=\frac{2 n_{k-1} \cos \left(\theta_{k-1}\right)}{n_{k-1} \cos \left(\theta_{k}\right)+n_{k} \cos \left(\theta_{k-1}\right)} \\ r_{k}^{p}=\frac{n_{k-1} \cos \left(\theta_{k-1}\right)-n_{k} \cos \left(\theta_{k}\right)}{n_{k-1} \cos \left(\theta_{k-1}\right)+n_{k} \cos \left(\theta_{k}\right)} & t_{k}^{p}=\frac{2 n_{k-1} \cos \left(\theta_{k-1}\right)}{n_{k-1} \cos \left(\theta_{k-1}\right)+n_{k} \cos \left(\theta_{k}\right)}\end{array}$

In the case of normal incidence $\theta_{k}=0$, the values of the Fresnel coefficients are independent to the polarization condition [16]:

$r_{k}^{s}=r_{k}^{p}=\frac{n_{k-1}-n_{k}}{n_{k-1}-n_{k}} \quad t_{k}^{s}=t_{k}^{p}=\frac{2}{n_{k-1}-n_{k}}$

For $\theta=0$, the dephasing is of the form:

$\varphi_{k}=\frac{2 \cdot \pi \cdot n_{k}}{\lambda} \cdot d_{k}$

However, these quantities are generally complex. The coefficients of reflection and transmission are determined assuming that:

$E_{0}^{-}=E_{N+1}^{,-}=0$

The energy conservation equation gives:

$|r|^{2}+|t|^{2}=1$

Consequently,

$R=|r|^{2}$ And $\mathrm{T}=|t|^{2}$

\section{Reflection and transmission at the interface between two homogeneous media}

Let's consider an interface between two semi-infinite media (an environment of index $\tilde{n} 1$ and an isotropic material with complex index $\tilde{\mathrm{n}} 2$ as shown in Figure 2. The refractive index is defined by its real part $\mathrm{n} 2$ and its imaginary part K2, respectively called the refractive index and extinction coefficient.

$\tilde{\mathrm{n}} 2=\mathrm{n} 2-\mathrm{i} \mathrm{k} 2$

For a normal incident angle: 
$R=R_{T E}=R_{T M}=\left[\frac{\tilde{n}_{1}-\tilde{n}_{2}}{\tilde{n}_{1}+\tilde{n}_{2}}\right]^{2} \quad T=T_{T E}=T_{T M}=\left(\frac{2 \tilde{n}_{1}}{\tilde{n}_{1}+\tilde{n}_{2}}\right)^{2} \frac{\tilde{n}_{2}}{\tilde{n}_{1}}$

For example, in the case of Silicon $(\mathrm{Si})$, the dioptre air-silicon gives:

$\tilde{\mathrm{n}} 1=1$ and $\tilde{\mathrm{n}} 2=\mathrm{n}_{\mathrm{Si}}-\mathrm{i} \mathrm{k}_{\mathrm{Si}}$

The reflection coefficient becomes:

$R=\frac{\left(1-n_{s i}\right)^{2}+k_{s i}^{2}}{\left(1+n_{s i}\right)^{2}+k_{s i}^{2}}$

\section{Modeling of the anti-reflection layer}

To reduce or cancel the reflections, the condition of destructive interference (r12=-r21) is required. This relation is called the principle of layers' antireflection [13]. When the dephasing is $\varphi= \pm \pi$, the expression of the thickness of the layer is obtained through:

$\widetilde{\mathrm{n}_{1}}=1 \quad \mathrm{~d}=\lambda / 4 \mathrm{n}_{2}$

\begin{tabular}{|lc|}
\multicolumn{1}{|l|}{$\mathrm{n}_{0}=1$} & Air \\
\hline $\mathrm{n}_{1}$ & \\
& Anti-reflection layer \\
\hline $\mathrm{n}_{2}$ & Selective emitter \\
& (Semi-conductor layer) \\
\hline
\end{tabular}

Fig. 4: A Multilayered Structure Consisting of an Antireflection Layer (Glass, Zno) and a Selective Emitter (Si).

\section{Results and discussion}

A SCILAB program was developed to assess the various radiative parameters. The results are presented in this section. 6.1. Influence of the extinction index for normal incidence

In practice, to measure the reflection coefficient in the case of low absorption, one side of a prism material is used. Indeed, the use of a parallel plate leads to multiple reflections with interferences. In the case of high absorption, one can just use a blade of sufficient thickness to completely absorb the transmitted wave.

Fig. 5 presents the variation of the reflection coefficient with the extinction index for air and various semiconductors $(\mathrm{ZnO}, \mathrm{SiC}, \mathrm{Si}, \mathrm{Ge})$.

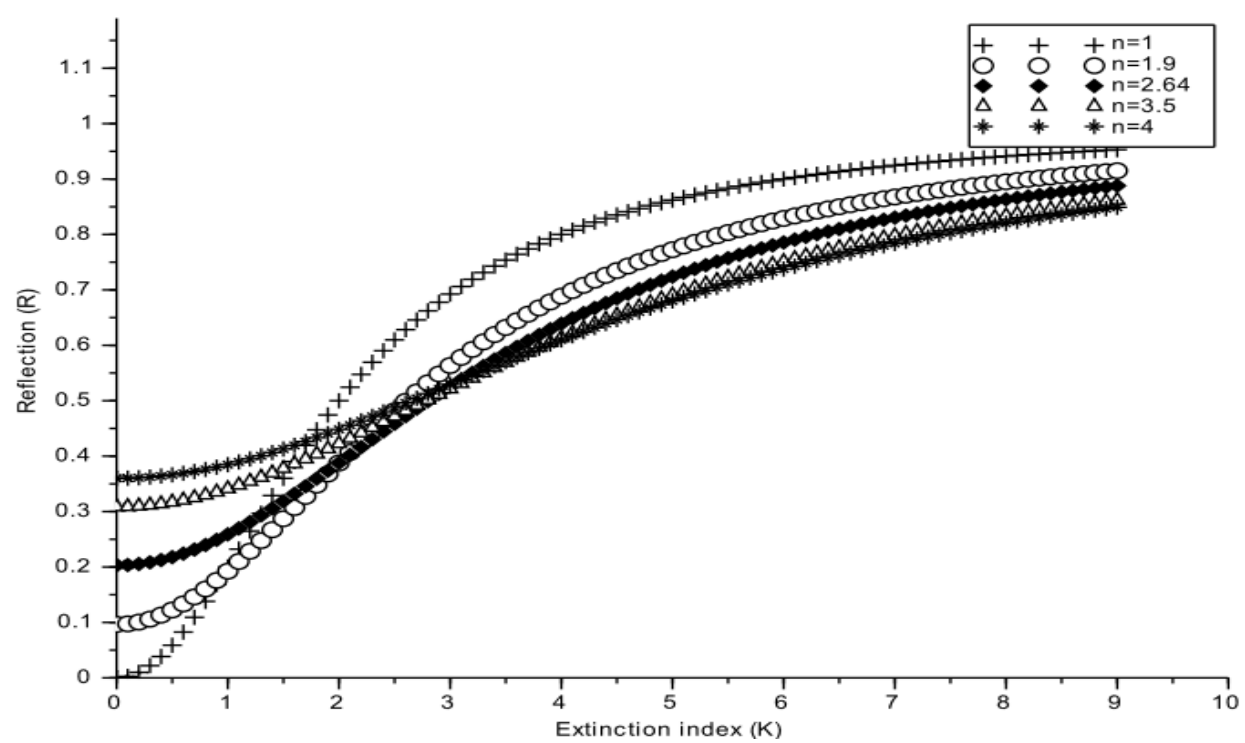

Fig. 5: Variation of the Reflection Coefficient with the Extinction Index for Air and Some Semi-Conductors (Zno, Sic, Si, Ge). 
As shown in fig. 5 and table 1, it could be seen that the reflection coefficient is highly influenced by the extinction index $(\mathrm{K})$. According to the type of the material, the reflection coefficient range from 0.1 to 0.9 . For an extinction index of 2.6, the materials $(\mathrm{ZnO}, \mathrm{SiC}, \mathrm{Si}, \mathrm{Ge})$ have practically the same reflection coefficient $(\mathrm{R}=0.5)$. In practice the best suited material should have little reflection coefficient, so SiC, Si, and Ge may be used in the TPV and TPX systems. In addition, the Germanium could support very high temperature compared to the two other.

Table 1: Influence of the Extinction Index (K) Corresponding to Each Material.

\begin{tabular}{llllll}
\multicolumn{7}{c}{ Table 1: Influence of the Extinction Index (K) Corresponding to Each Material. } \\
\hline Material & Air & ZnO & SiC & Si & Ge \\
\hline Normal index of refraction (n) & 1 & 1.9 & 2.64 & 3.5 & 4 \\
Range of the reflection coefficient & $0-0.95$ & $0.1-0.9$ & $0.2-0.78$ & $0.3-0.76$ & $0.35-0.75$ \\
\hline
\end{tabular}

\subsection{Influence of the ratio $(\mathrm{e} / \lambda)$ on the reflection, transmission and absorption coefficients}

\subsubsection{Case of low absorption $(K<1)$}

In this case, the optical properties are mainly influenced by the index $\mathrm{n}$, which is different to 1 .

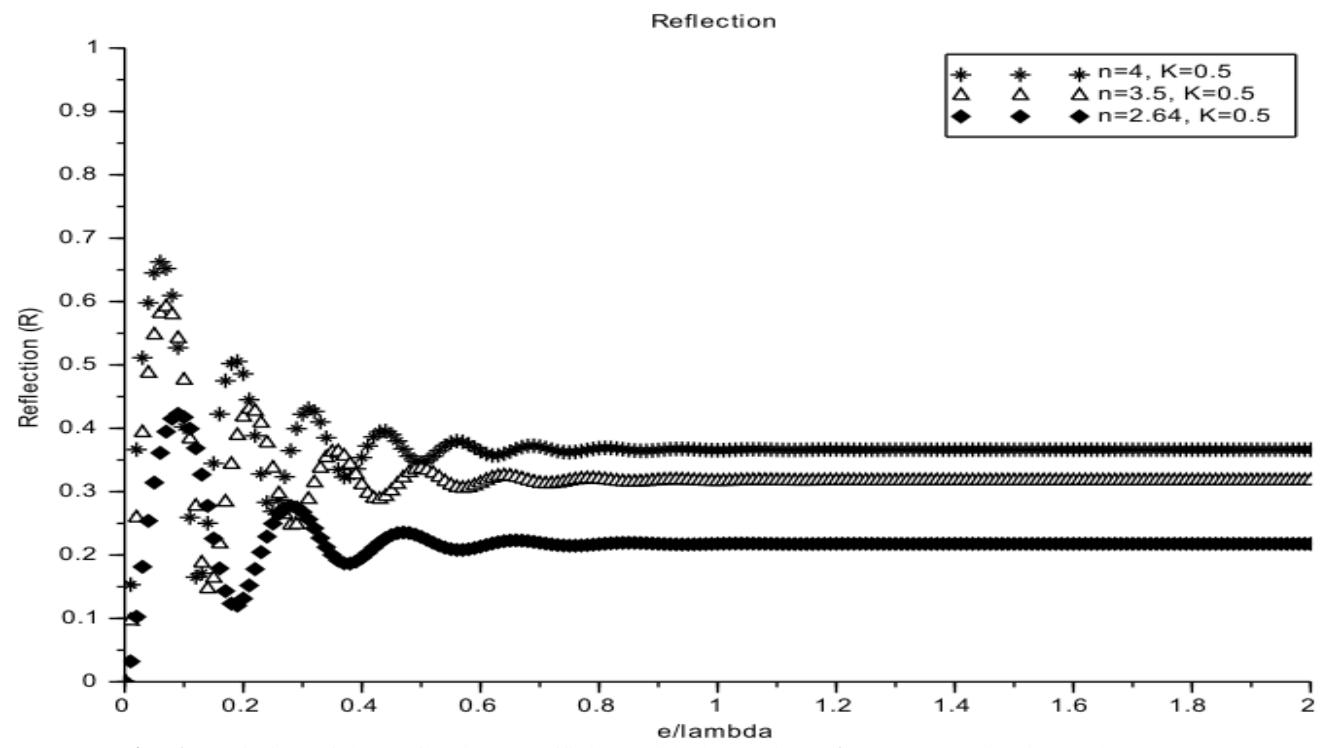

Fig. 6: Variation of the Reflection Coefficient with the Ratio (E/ $\lambda$ ) For an Extinction Index $\mathrm{K}=0.5$.

Figure 6 presents the variation of reflection coefficient with the ratio (e/ $\lambda$ ) for an extinction index $\mathrm{K}=0.5$ in the case of low absorption for the germanium $(\mathrm{Ge})$, the Silicon $(\mathrm{Si})$ and the Silicon carbide ( $\mathrm{SiC})$. It could be seen from fig. 6 and table 2 that in the case of low absorption, the reflection coefficient fluctuates for $0 \leq \mathrm{e} / \lambda \leq 0.6$ and more constant for $\mathrm{e} / \lambda>0.6$. In addition, the $\mathrm{SiC}$ has interesting reflection coefficient compared to the two others.

\begin{tabular}{llll}
\multicolumn{2}{c}{ Table 2: Range of the Reflection Coefficient in the Case of Low Absorption for Some Materials (Ge, Si, Sic). } \\
\hline Material & Ge & $\mathrm{Si}$ & $\mathrm{SiC}$ \\
\hline Reflection coefficient $(\mathrm{R})$ & $0-0.38$ & $0-0.35$ & $0-0.2$ \\
Ratio $(\mathrm{e} / \boldsymbol{\lambda})$ & $0-0.78$ & $0-0.75$ & $0-0.7$ \\
\hline
\end{tabular}

Figure 7 presents the variation of the transmission coefficient of some semi-conductors $(\mathrm{Ge}, \mathrm{Si}, \mathrm{SiC})$ with the ratio (e/ $\lambda$ ) in the case of low absorption for an extinction coefficient $\mathrm{K}=0.5$. 


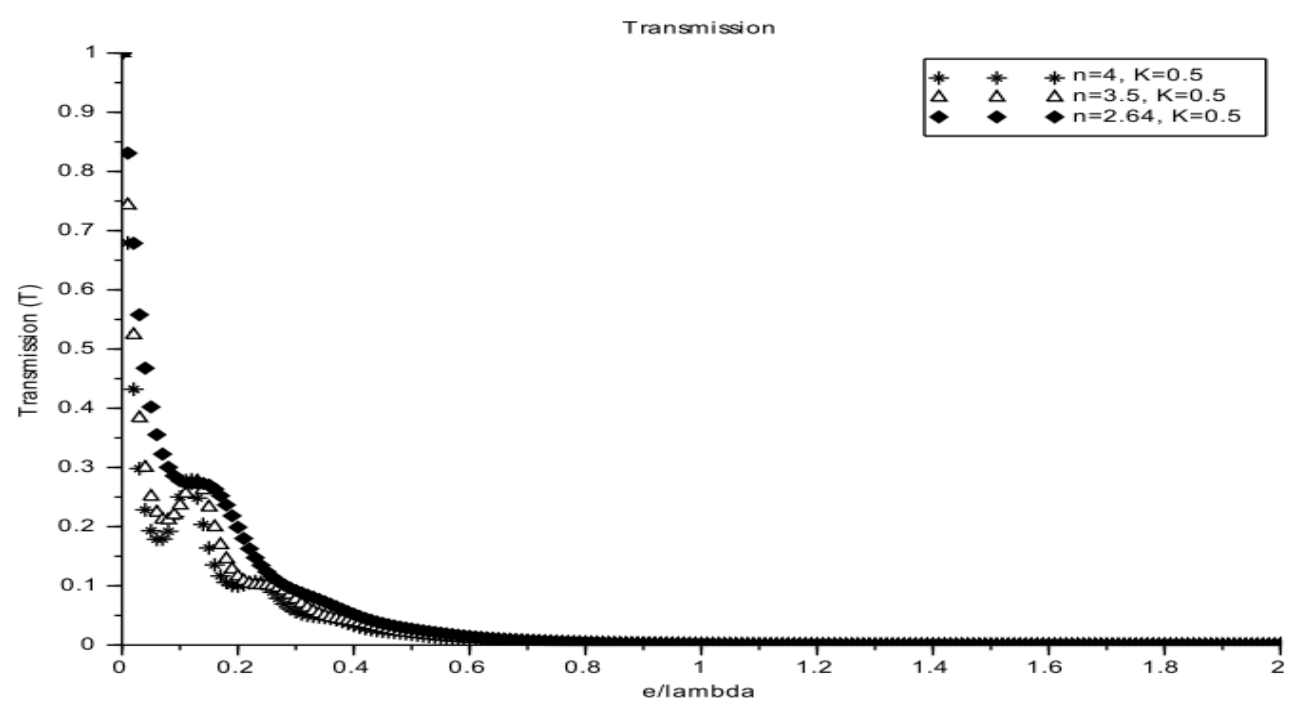

Fig. 7: Variation of the Transmission Coefficient of Some Semi-Conductors (Ge, Si, Sic) with the Ratio (E/ $/$ ) in the Case of Low Absorption for an Extinction Coefficient $\mathrm{K}=0.5$.

It could be seen from fig. 7 and table 3 that in the case of low absorption, the transmission coefficient fluctuates for $0 \leq \mathrm{e} / \lambda \leq 0.6$ and more constant for $\mathrm{e} / \lambda>0.6$. From the same table, it is seen that the ranges of the transmission coefficient and thicknesses are practically the same.

In general, for TPV and TPX systems, layers with large thickness make that the transmitted wave is not completely absorbed by the material. So there will not be a good heat exchange between the emitter and the TPV or TPX cell.

Table 3: Range of the Transmission Coefficient in the Case of Low Absorption for Some Semi-Conductors (Ge, Si, Sic).

\begin{tabular}{llll}
\hline Material & Ge & $\mathrm{Si}$ & $\mathrm{SiC}$ \\
\hline Transmission coefficient $(\mathrm{T})$ & $0-0.9$ & $0-0.9$ & $0-0.9$ \\
Ratio $(\mathrm{e} /(\lambda)$ & $0-0.8$ & $0-0.8$ & $0-0.8$ \\
\hline
\end{tabular}

Figure 8 shows the variation of the absorption coefficient of some semi-conductors $(\mathrm{Ge}, \mathrm{Si}, \mathrm{SiC})$ with the ratio (e/ $\lambda$ ) in the case of low absorption for an extinction coefficient $\mathrm{K}=0.5$.

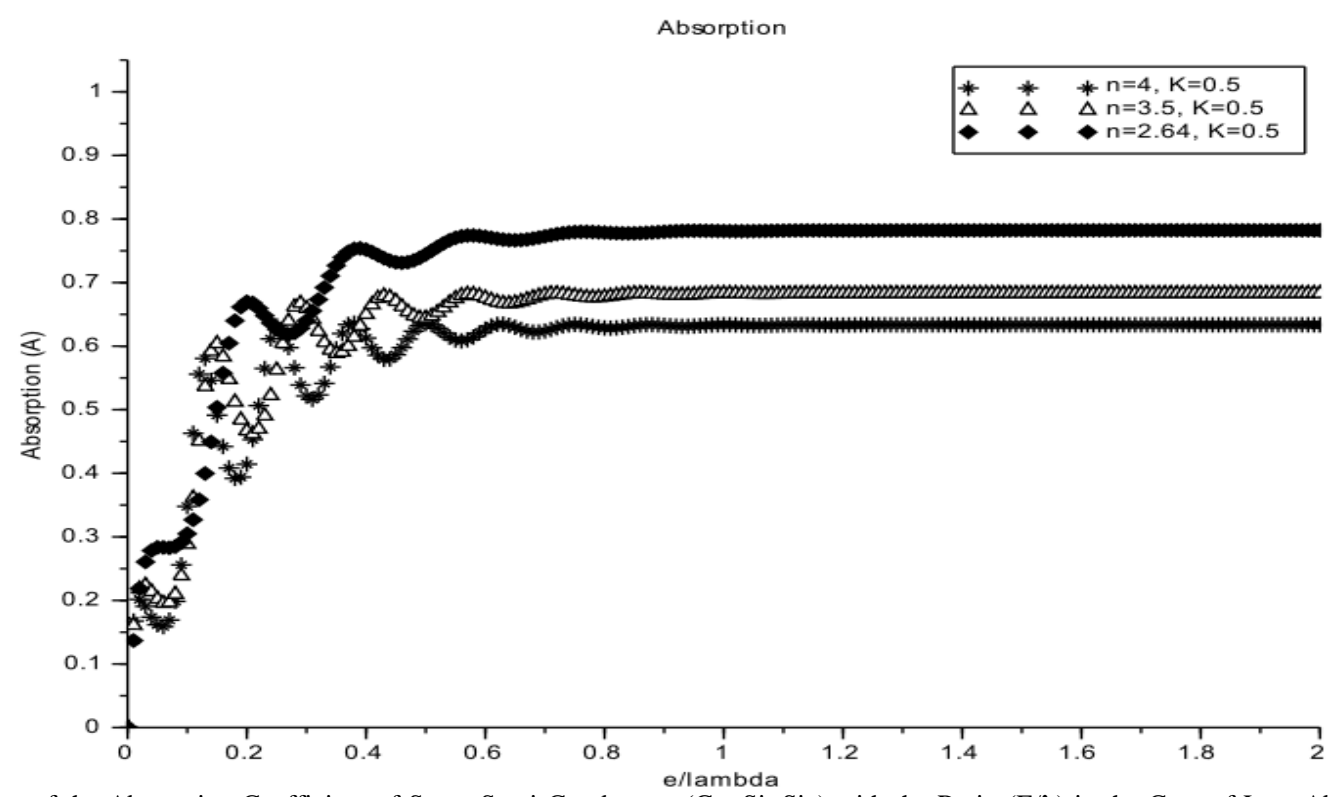

Fig. 8: Variation of the Absorption Coefficient of Some Semi-Conductors (Ge, Si, Sic) with the Ratio (E/ $\lambda$ ) in the Case of Low Absorption for an Extinction Coefficient $\mathrm{K}=0.5$.

It could be seen from fig. 8 and table 4 that in the case of low absorption, the absorption coefficient fluctuates for $0 \leq \mathrm{e} / \lambda \leq 0.6$ and more constant for e/ $\lambda>0.6$. It is seen from table 4 that $\mathrm{SiC}$ and $\mathrm{Si}$ present interesting characteristics. In fact, low absorptivity yields to poor performance of the TPV and TPX systems. The problem is most of the time solved by a specific antireflection treatment as presented in section 6.3. 
Table 4: Range of Absorption Coefficient of Some Semi-Conductors (Ge, Si, Sic) in the Case of Low Absorption.

\begin{tabular}{llll}
\hline Material & Ge & Si & SiC \\
\hline Range of absorption coefficient (A) & $0.05-0.63$ & $0.05-0.65$ & $0.05-0.75$ \\
Ratio $(\mathrm{e} / \boldsymbol{\lambda})$ & $0-0.85$ & $0-0.85$ & $0-0.75$ \\
\hline
\end{tabular}

\subsubsection{Case of high absorption $(K>1)$}

Unlike the case of low absorption which causes multiple oscillations, high absorption generates fewer oscillations. In this case, the extinction index is greater than one.

Figure 9 shows the variation of the reflection coefficient of some semi-conductors $(\mathrm{Ge}, \mathrm{Si}, \mathrm{SiC})$ with the ratio (e/ $\lambda$ ) in the case of high absorption for an extinction coefficient $\mathrm{K}=5$.

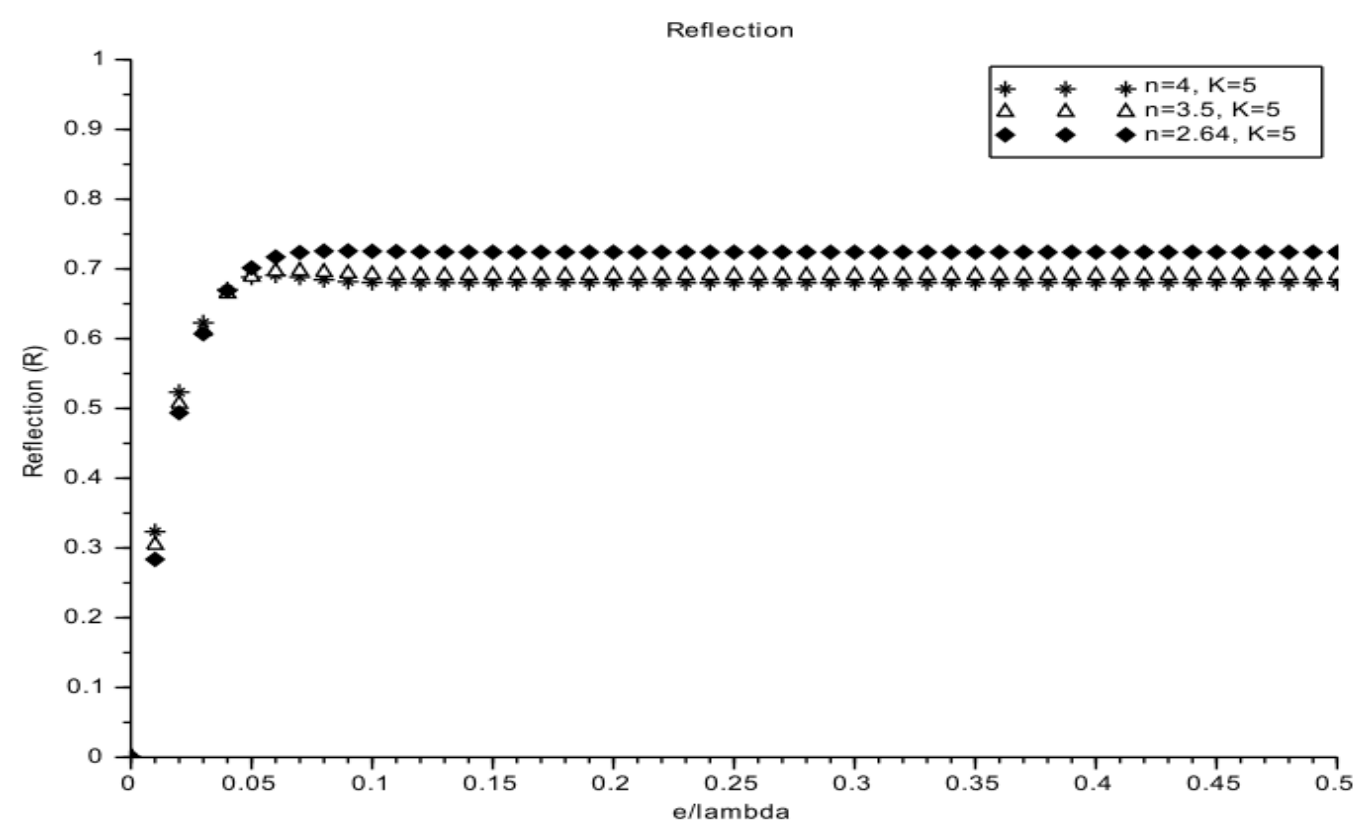

Fig. 9: Variation of the Reflection Coefficient of Some Semi-Conductors (Ge, Si, Sic) with the Ratio (E/ $\lambda$ ) in the Case of High Absorption for an Extinction Coefficient $\mathrm{K}=0.5$.

It could be seen from fig. 9 and table 5 that in the case of high absorption, the reflection coefficient fluctuates for $0 \leq \mathrm{e} / \lambda \leq 0.06$ and more constant for $\mathrm{e} / \lambda>0.06$. In this case, reflection does not present a minimum. So, TPV and TPX systems with this kind of reflection will have a low absorption, consequently a low efficiency.

Table 1: Range of Reflection Coefficient of Some Semi-Conductors (Ge, Si, Sic) in the Case of High Absorption.

\begin{tabular}{|c|c|c|c|}
\hline Material & $\mathrm{Ge}$ & $\mathrm{Si}$ & $\mathrm{SiC}$ \\
\hline Range of reflection coefficient $(\mathrm{R})$ & $0-0.68$ & $0-0.7$ & $0-0.73$ \\
\hline Ratio $(\mathrm{e} / \lambda)$ & $0-0.08$ & $0-0.07$ & $0-0.05$ \\
\hline
\end{tabular}

Figure 10 shows the variation of the transmission coefficient of some semi-conductors $(\mathrm{Ge}, \mathrm{Si}, \mathrm{SiC})$ with the ratio (e/ $\lambda$ ) in the case of high absorption for an extinction coefficient $\mathrm{K}=5$. 


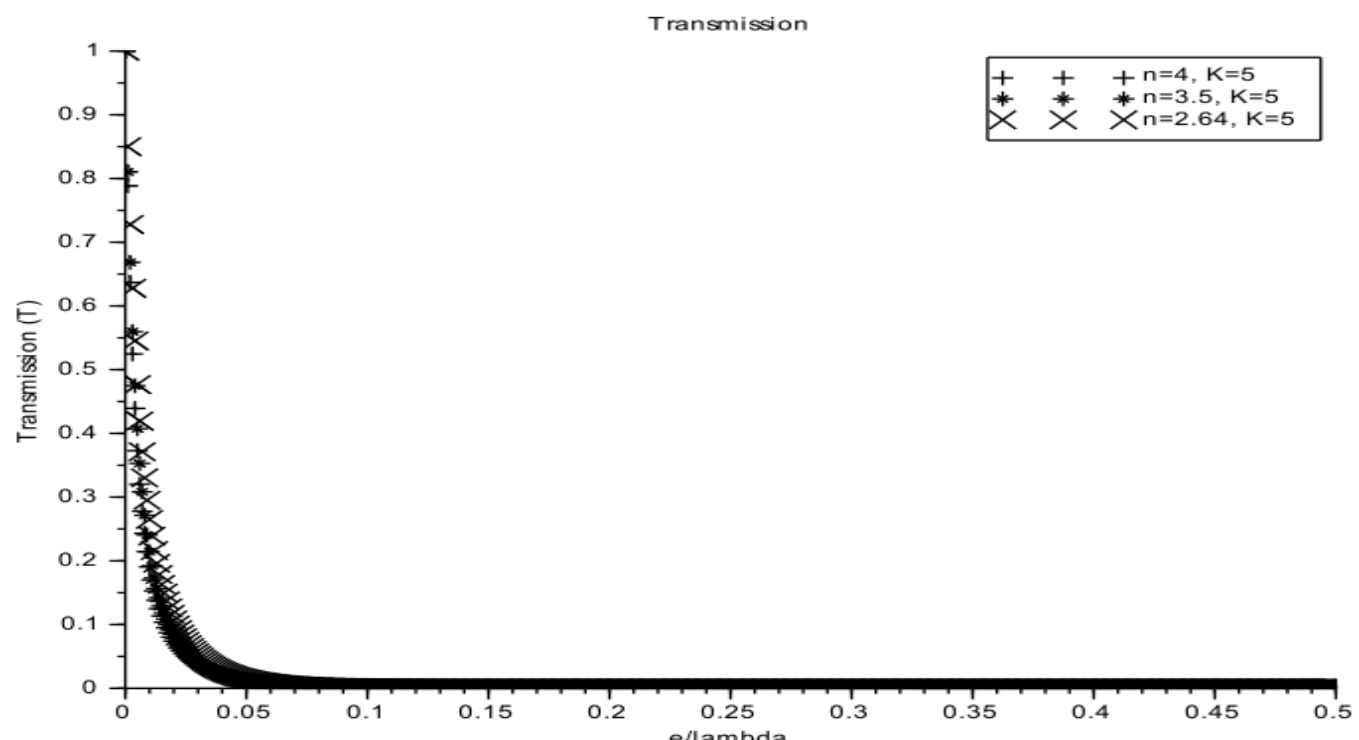

Fig. 10: Variation of the Transmission Coefficient of Some Semi-Conductors (Ge, Si, Sic) with the Ratio (E/ $/$ ) in the Case of High Absorption for an Extinction Coefficient $\mathrm{K}=5$.

It could be seen from fig. 10 and table 6 that in the case of high absorption, the transmission coefficient fluctuates for $0 \leq \mathrm{e} / \lambda \leq 0.06$ and more constant for $\mathrm{e} / \lambda>0.06$. The same remarks as in the case of low absorption are made.

Table 6: Range of Transmission Coefficient of Some Semi-Conductors (Ge, Si, Sic) in the Case of High Absorption

\begin{tabular}{llll}
\hline Material & $\mathrm{Ge}$ & $\mathrm{Si}$ & $\mathrm{SiC}$ \\
\hline Range of transmission coefficient $(\mathrm{T})$ & $0-0.8$ & $0-0.8$ & $0-0.8$ \\
Ratio $(\mathrm{e} / \lambda)$ & $0-0.09$ & $0-0.09$ & $0-0.09$ \\
\hline
\end{tabular}

Figure 11 shows the variation of the absorption coefficient of some semi-conductors $(\mathrm{Ge}, \mathrm{Si}, \mathrm{SiC})$ with the ratio (e/ $\lambda$ ) in the case of high absorption for an extinction coefficient $\mathrm{K}=5$.

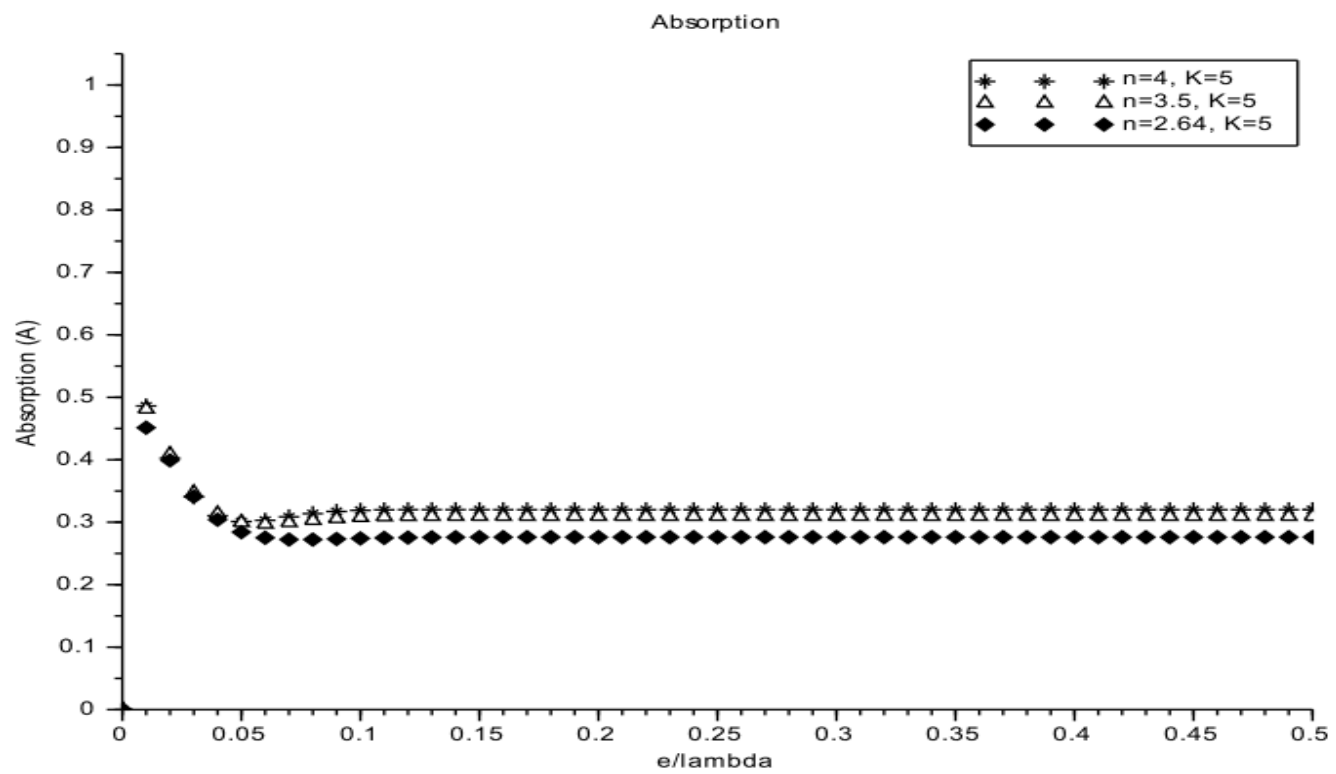

Fig. 11: Variation of the Absorption Coefficient of Some Semi-Conductors (Ge, Si, Sic) with the Ratio (E/ $\lambda$ ) in the Case of High Absorption for $n$ Extinction Coefficient $\mathrm{K}=5$.

It could be seen from fig. 11 and table 7 that in the case of high absorption, the absorption coefficient fluctuates for $0 \leq$ $\mathrm{e} / \lambda \leq 0.06$ and more constant for $\mathrm{e} / \lambda>0.06$. The results are in conformity with the law of Kirchhoff which says that a good absorber is also a good emitter. Systems operating in this condition present a good efficiency. 
Table 7: Range of Absorption Coefficient of Some Semi-Conductors (Ge, Si, Sic) in the Case of High Absorption.

\begin{tabular}{llll}
\hline Material & Ge & Si & SiC \\
\hline Range of absorption coefficient (A) & $0.11-0.32$ & $0.11-0.31$ & $0.11-0.30$ \\
Ratio $(\mathrm{e} / \lambda)$ & $0-0.07$ & $0-0.06$ & $0-0.05$ \\
\hline
\end{tabular}

\subsection{Antireflection treatment at the interface}

To minimize multiple reflections, an antireflective treatment is sometimes needed. In this study we will consider the case of a glass and the $\mathrm{ZnO}$.

\subsubsection{Case of a glass layer}

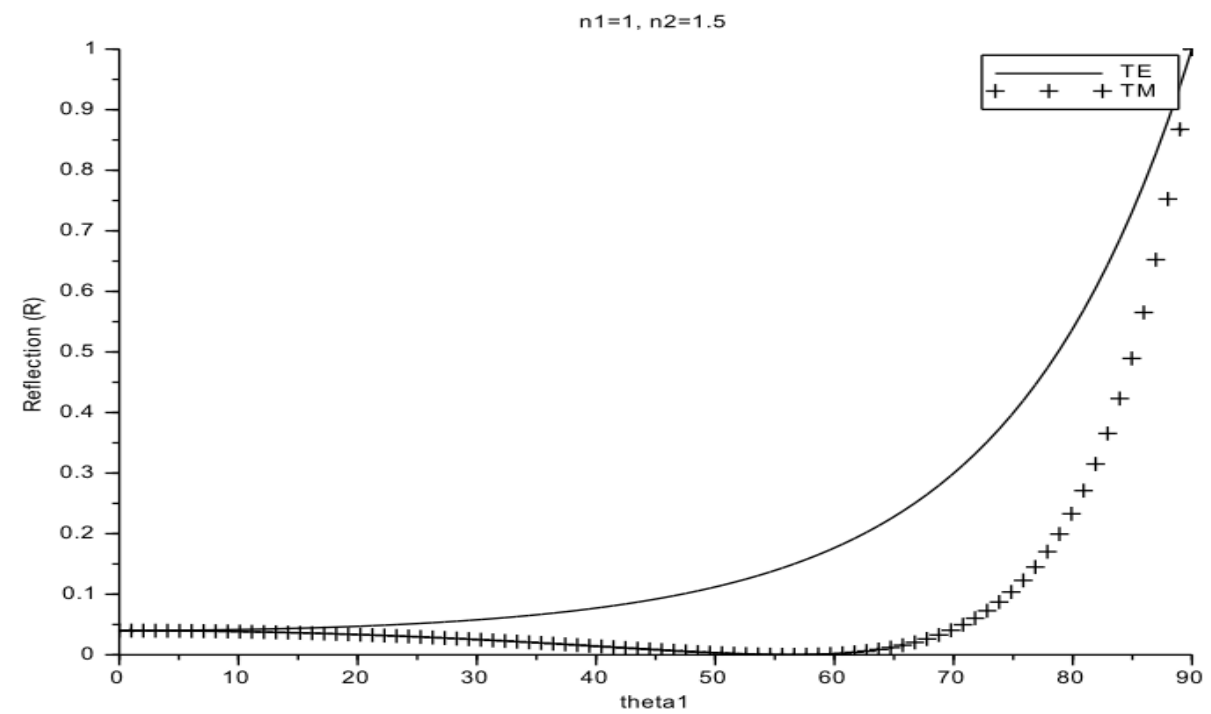

Fig. 12: Fresnel Factors of Reflection for a Radiative Energy Reflected on the Surface of Silicon in the Visible Range Using a Glass Layer as Antireflection Treatment.

In Figure 12, the plain curve represents the TE polarization, and the dot curve the TM polarization. In TM polarization, there is practically a cancellation of reflection at the brewsterian angle $56.3^{\circ}$; the reflection coefficient here has a value $\mathrm{R}=0.04$. When $\theta \mathrm{i}$ tends to $90^{\circ}$, the two coefficients tend to 1 .

Fig. 13 presents the case of a treatment using a glass antireflective layer with a thickness equals to the quarter of wavelength value. It could be seen that in this case, the reflectance is zero for the two polarizations (TM and TE) at a Brewster angle of $56^{\circ}$ in contrast to the case presented in figure 12 where there a cancellation reflectance only in TM polarization. Reflection in this case is reduced to $\mathrm{R}=0.01$.

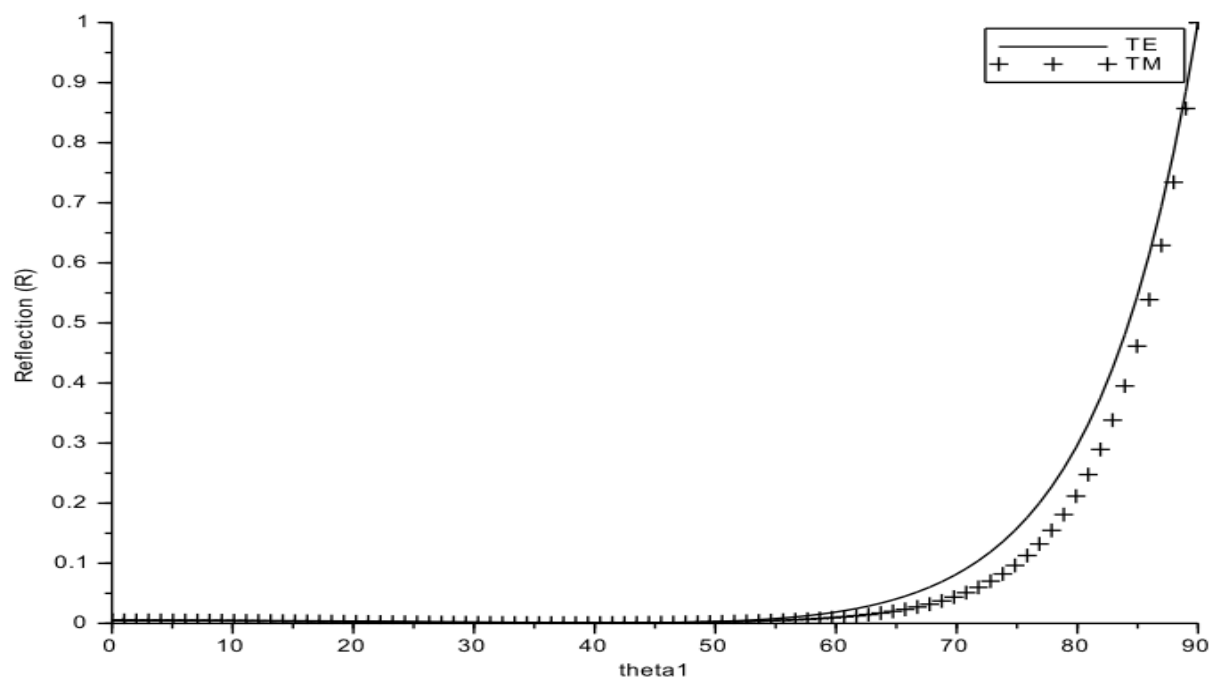

Fig. 13: Representation of the Antireflection Curve for a Thickness Equals to the Quarter of the Wavelength Value in the Case of an Antireflective Treatment with Glass. 


\subsubsection{Case of zinc oxide $(\mathrm{ZnO})$}

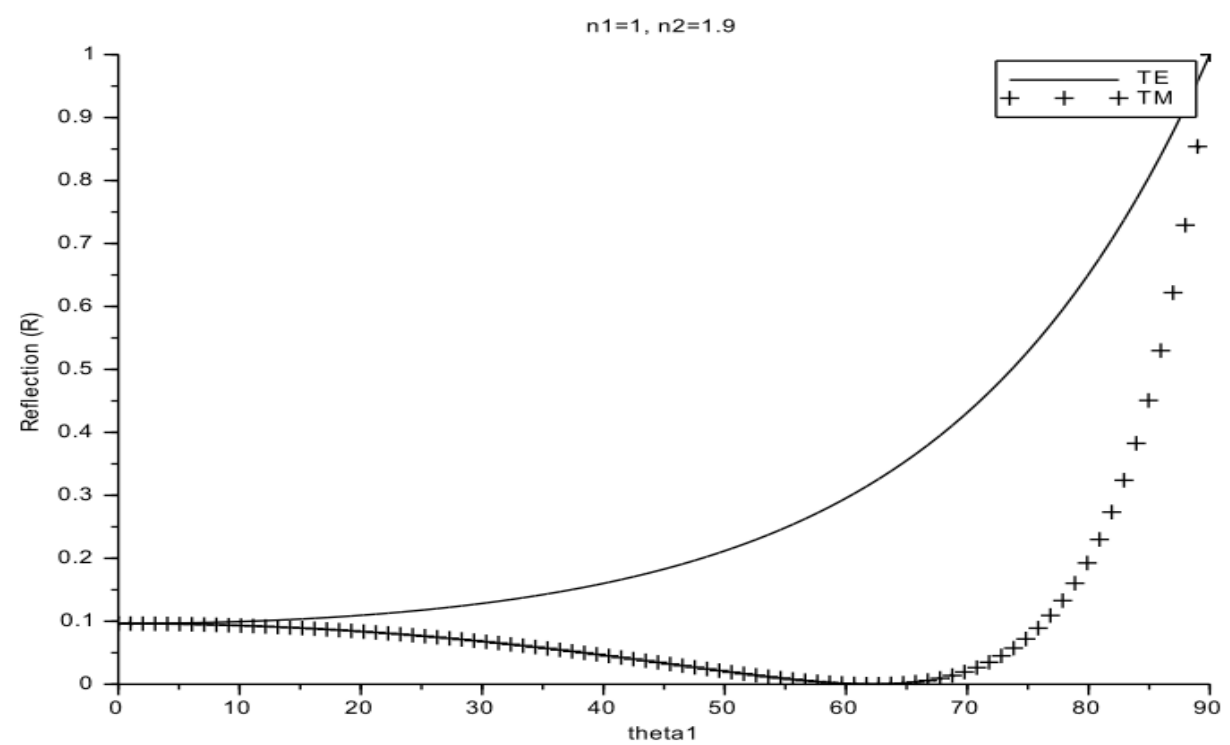

Fig. 14: Fresnel Factors of Reflection for a Radiative Energy Reflected on the Surface of Silicon in the Visible Range Using a Zinc Oxide as Antireflection Treatment.

In Figure 14, the plain curve represents the TE polarization, and the dot curve the TM polarization. In TM polarization, it could be observed a cancellation of reflection at a brewsterian angle $\theta_{B}=62.24^{0}$ where the reflection coefficient's value is $\mathrm{R}=0.1$

Fig. 15 presents the case of a treatment using a $\mathrm{ZnO}$ antireflective layer with a thickness equals to the quarter of wavelength value. Here, there is no cancellation of the reflectance at the two polarizations (TM and TE) at the Brewster angle. The reflectance value is $\mathrm{R}=0.03$

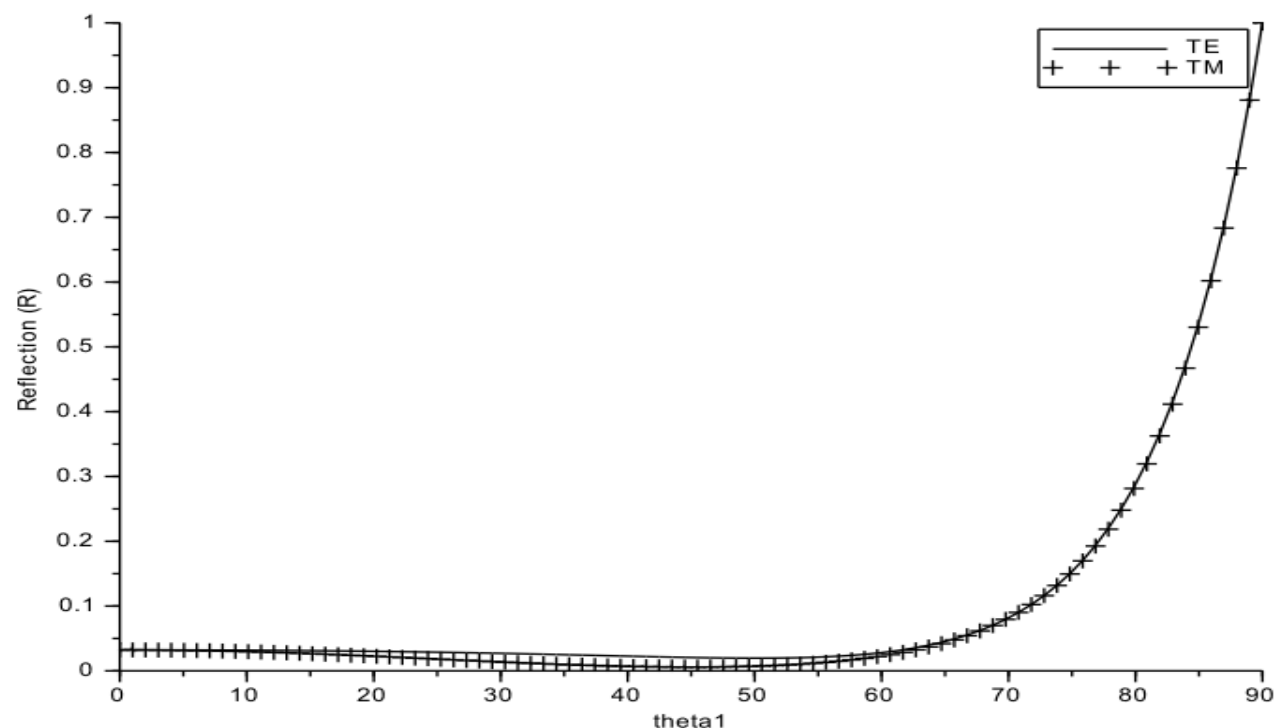

Fig. 15: Representation of the Antireflection Curve in for A Thickness Equals to the Quarter of the Wavelength Value in the Case of an Antireflective Treatment with $\mathrm{ZnO}$.

Table 8 present a brief summary of the anti-reflective treatment. It could be seen that using glass for an antireflective treatment is better than $\mathrm{ZnO}$.

Table 8: Summary of the Parameters of the Antireflection Treatment.

\begin{tabular}{lll}
\hline Material & ZnO & Glass \\
\hline Index of the material $\left(\mathrm{n}_{\mathrm{s}}\right)$ & 1.9 & 1.5 \\
Ideal Index of the material $\left(\mathrm{n}_{1}\right)$ & 1.37 & 1.22 \\
Untreated Reflectance $(\mathrm{R})$ & 0.1 & 0.04 \\
Treated Reflectance $(\mathrm{R})$ & 0.03 & 0.013 \\
\hline
\end{tabular}




\section{Conclusion}

In this paper we presented an assessment of the radiative properties of some semi-conductors that can be used in TPV and TPX systems. The method was based on the principles of thermal radiation, Fresnel's theories of radiation, and Maxwell's equations describing the classical theory of the propagation of electromagnetic waves in a homogeneous and isotropic medium. The influence of the extinction index on the optical characteristics of some materials $(\mathrm{SiC}, \mathrm{Si}, \mathrm{Ge}$, and $\mathrm{ZnO}$ ) was evaluated. The transfer matrix method implemented in under a scilab platform was used to evaluate and assess the behaviour of the radiative properties of strongly and weakly absorbing multilayered structures. It was found that the use of a non-reflecting treatment (glass or $\mathrm{ZnO}$ ) on the material, enables to reduce multiple reflections and therefore to improve the efficiency of the system.

Nomenclature

$D_{k}:$ Stamp passage of the $\mathrm{k}^{\text {th }}$ layer

$d_{k}:$ Thickness of the $\mathrm{k}^{\text {th }}$ layer $(\mu \mathrm{m})$

$\vec{E}$ : Electric field

$E_{k}$ : Electric field of the $\mathrm{k}^{\text {th }}$ layer

$E_{k}^{s}$ : Electric field in parallel polarization of the $\mathrm{k}^{\text {th }}$ layer

$E_{k}^{p}$ : Electric field in perpendicular polarization of the $\mathrm{k}^{\text {th }}$ layer

$e:$ Thickness

$K:$ Index of extinction

$k$ : Amplitude of the vector of wave

$L^{0}$ : Total Brightness

$L_{\lambda}^{0}:$ Spectral brightness

$L_{\lambda}^{\prime 0}$ : Spectral brightness in the vacuum

$M_{\lambda m}^{0}$ : Monochromatic flux density total

$M_{\lambda}^{0}$ : Monochromatic flux density

$n:$ Index of refraction

$n_{k}:$ Index of refraction of the $\mathrm{k}^{\text {th }}$ layer

$p_{k}$ : Stamp propagation of the $\mathrm{k}^{\text {th }}$ layer

$\mathrm{R}$ : Factor of reflection (\%)

$r(\lambda, \theta, \phi)$ : Coefficient of directional reflectivity

$r_{k}^{s}$ : Reflection in parallel polarization of the $\mathrm{k}^{\text {th }}$ layer

$r_{k}^{p}:$ Reflection in perpendicular polarization of the $\mathrm{k}^{\text {th }}$ layer

$r$ : Coefficient of reflection of Fresnel (\%)

$\mathrm{T}$ : Factor of transmission $(\%)$

$\mathrm{T}$ : Temperature $(\mathrm{K})$

$t_{k}^{s}$ : Transmission in parallel polarization of the $\mathrm{k}^{\text {th }}$ layer

$t_{k}^{p}$ : Transmission in perpendicular polarization of the $\mathrm{k}^{\text {th }}$ layer

Greek symbols

$\theta_{l}:$ Limit angle $\left(^{\circ}\right)$

$\theta_{B}:$ Brewster's angle $\left({ }^{\circ}\right)$

$\theta$ : Angle of incidence

$\theta_{k}:$ Angle of incidence of the $\mathrm{k}^{\text {th }}$ layer

$\lambda$ : Wavelength $(\mu \mathrm{m})$

$\tau:$ Coefficient of transmission of Fresnel (\%)

$\sigma:$ Conductivity of the medium

$\varepsilon(\lambda, \theta, \phi):$ Directional and monochromatic emissivity

$\tau(\lambda, \theta, \phi)$ : Coefficient of directional transmissivity 


\section{References}

[1] K.R. Catchpole, K.L. Lin, M.A. Green, A.G. Aberle, R. Corkish, J. Zhao, A. Wang. Thin semiconducting layers and nanostructures as active and passive emitters for thermophotonics and thermophotovoltaics. Physica E 14 (2002) 91-95. http://dx.doi.org/10.1016/S1386-9477 (02)00363-6.

[2] Thomas Bauer (2011). Thermophotovoltaics: Basic Principles and Critical Aspects of System Design. Springer-Verlag Berlin Heidelberg. http://dx.doi.org/10.1007/978-3-642-19965-3.

[3] Donald L. Chubb. Fundamentals of Thermophotovoltaic energy conversion. NASA Glenn Research Center 1000 Brookpark Road, MS 302-1 Cleveland, OH 44135 USA. Published by Elsevier B.V. 2007.

[4] H.H. Kolm, Quarterly Progress Report, Solid State Research, Group 35, MIT-Lincoln Laboratory, Lexington, MA, May 1, $1956,13$.

[5] Green Martin A. (2000). "'Third Generation Photovoltaics: Advanced Structures Capable of High Efficiency at Low Cost". Proceedings of the 16th European Photovoltaic Solar Energy Conference. Glasgow, Scotland

[6] Bitnar, B. (2003). "Silicon, germanium and silicon/germanium photocells for thermophotovoltaics applications". Semiconductor Science and Technology 18 (5): S221. Bibcode2003SeScT.18S.221B http://dx.doi.org/10.1088/0268-1242/18/5/312.

[7] Jérémie Drevillon and Philippe Ben-Abdallah. Abinitio design of coherent thermal sources. Journal of Applied Physics, 102(11):114305, 2007 http://dx.doi.org/10.1063/1.2816244

[8] E. Nefzaoui, J. Drevillon, and K. Joulain. Conception ET optimisation d'émetteurs sélectifs pour applications thermophotovoltaïques. Institut Pprime, CNRS-Université de Poitiers-ENSMA, Département fluide, Thermique, Combustion 2010.

[9] Jean-Jacques Greffet, Remi Carminati, Karl Joulain, Jean-Philippe Mulet, Stephane Mainguy, and Yong Chen. Coherent emission of light by thermal sources. Nature, 416:61-64, 2002 http://dx.doi.org/10.1038/416061a.

[10] A. M. Portis, Electromagnetic Fields, Sources and Media, John Wiley \& Sons, 1978, Chapter 12.

[11] K. Lagha-Menouer. Etude ET réalisation d'une cellule solaire multi couches du type Si-SiO2-SnO2-ZnO par APCVD. Doctorat en Electronique. Universite Mouloud Mammeri de Tizi-Ouzou (2011)

[12] O. Perrot. I.U.T. de Saint-Omer Dunkerque, Département Génie Thermique et énergie, COURS DE RAYONNEMENT 3ème Semestre (20102011). 